\title{
Comparison of a generic and a rhinitis-specific quality-of-life (QOL) instrument in patients with house dust mite allergy: relationship between the SF-36 and Rhinitis QOL Questionnaire
}

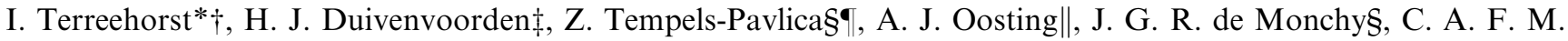 \\ Bruijnzeel-Koomen $\|$ and R. Gerth van Wijk* \\ *Department of Allergology and †Department of Internal Medicine, Erasmus Medical Centre, Rotterdam, The Netherlands, $\ddagger$ Institute of Medical \\ Psychology and Psychotherapy, NIHES, Erasmus University, Rotterdam, The Netherlands, §Department of Allergology, University Hospital, Groningen, \\ The Netherlands, -Department of Internal Medicine, Albert Schweitzer Hospital, Dordrecht, The Netherlands and ||Department of Dermatology, \\ University Medical Centre, Utrecht, The Netherlands
}

\begin{abstract}
Summary
Background Generic and disease-specific quality-of-life (QOL) questionnaires are commonly used in subjects with allergic rhinitis (AR). AR, however, is closely associated with other disorders such as bronchial asthma and atopic dermatitis (AD). These co-morbid associations may have an effect on the inter-relation of generic and disease-specific QOL outcomes and the behaviour of this interrelation in time.

Objective To unravel the inter-relationships between the outcome of a generic instrument (SF-36) and a disease-specific instrument (Rhinitis QOL Questionnaire (RQLQ)).

Materials and methods In the framework of a randomized clinical trial with respect to the efficacy of impermeable bedding covers in house dust mite (HDM) allergy, SF-36 and RQLQ were administered to 224 adults with $\mathrm{AR}$ and/or allergic asthma and/or AD at baseline and after 12 months of intervention. Regression analysis and canonical correlation were used to estimate overlap. Results Overlap between SF-36 and RQLQ domains in terms of explained variance ranged from $6 \%$ to $56 \%$. Canonical correlation yielded low coefficients $(0.16-0.27)$. Moreover, both SF-36 and RQLQ scores did not change significantly during the intervention.

Conclusion In patients with HDM allergy characterized by co-morbid associations, SF-36 and RQLQ cover different aspects in QOL. It is advocated to use both simultaneously in performing QOL studies.
\end{abstract}

Keywords allergy, canonical correlation, redundancy analysis, rhinitis, RQLQ, SF-36 Submitted 19 January 2004; revised 18 May 2004; accepted 2 July 2004

\section{Introduction}

In the last decade, interest in the impact of atopic disease on quality of life (QOL) has grown. It is generally accepted that disease may hamper patients in their day-to-day functioning. As a consequence, instruments for measuring QOL have been developed, both generic and disease specific [1-6]. In allergic rhinitis (AR), both types of questionnaires have been used to evaluate the burden of nasal symptoms [7-12]. Previous research indicated that rhinitis-related QOL is moderately associated with nasal symptoms and nasal hyper-reactivity [13].

In day-to-day practice, patients usually suffer from more than one atopic disorder. The rates of AR among asthmatics vary from $28 \%$ to $50 \%$ in studies published in the 1970 s and 1980s [14, 15]. Recently, in a population-based study the prevalence of rhinitis in patients with asthma was estimated at

Correspondence: I. Terreehorst, Department of Allergology, Erasmus Medical Centre, Dr Molewaterplein 40, 3015 GD Rotterdam, The Netherlands. E-mail: i.terreehorst@erasmusmc.nl
$78 \%$ [16]. Edfors-Lubs showed that the prevalence of rhinitis in patients with dermatitis was $29 \%$ [14]. In a previous study, we found that $92 \%$ of the asthmatic patients and $85 \%$ of the patients with atopic dermatitis (AD) had nasal symptoms [17].

Despite all evidence for the high co-morbidity in atopic disease, in daily clinical-care therapy focusses on the most predominant disease, i.e. asthma, AD or rhinitis.

Therefore, we recently evaluated the impact of different disorders (i.e. rhinitis, asthma and AD) and co-morbidity on QOL in general assessed by the SF-36 in adult patients with house dust mite (HDM) allergy [18]. This study was a part of a large multi-centre clinical trial to investigate the effect of impermeable bedding covers on symptoms of allergic asthma, AR and AD. Results indicated that these patients experienced impaired QOL. The (co)-existence of asthma, expressed in terms of diagnostic criteria or symptom severity, or the presence of sleep disorders as a consequence of AD impaired QOL even further. Because of the high prevalence of rhinitis, we could only evaluate the additional contribution of coexisting asthma and ADs on generic QOL. However, as both 
generic and disease-specific QOL have been assessed by questionnaires, this data set enabled us to investigate the inter-relationship between the generic SF-36 and the diseasespecific QOL questionnaire for rhinoconjunctivitis (Rhinitis QOL Questionnaire (RQLQ)) in a population characterized by rhinitis and concomitant allergic disorders. Moreover, the study design of the clinical trial permitted us to investigate the change of this overlap during 1 year of follow-up.

\section{Materials and methods}

\section{Study design and patients}

Patients took part in a multi-centre trial to study the effect of impermeable covers for bedding on complaints of atopic rhinitis, atopic asthma or AD or any combination of these conditions. Participating centres were the Allergology Departments of the University Hospitals of Groningen and Rotterdam and the Dermatology Department of the University Hospital Utrecht. The study was approved by the Medical Ethics Committees of all three University Hospitals and all patients gave written informed consent. Inclusion and exclusion criteria are described in Table 1.

After baseline measurements, including nasal provocation and lung function tests, patients were diagnosed as having atopic rhinitis, atopic asthma or AD according to preset criteria, described extensively elsewhere [18, 19].

Follow-up visits were scheduled 4 and 12 months after baseline measurements. All measurements were performed outside the pollen season to avoid interference.

\section{Randomization and blinding procedures}

The study was designed as a double-blind, placebo-controlled trial. The randomization procedure for all centres was performed by the Julius Centre for Patient Oriented Research. Each patient was assigned a research number. Research number and bedding measures were sent to the manufacturer of the encasings, HAL (Haarlem, the Netherlands). A carton box, containing all encasings, was sent to the research centre, with the research number written on the outside of the box. Patients took the box home and opened it in the absence of the research staff. Encasings for pillows, duvets and mattresses were applied after baseline measurements.

\section{Clinical measurements}

Generic QOL was measured by means of the SF-36. We used the translation of Aaronson of the MOS SF-36, originally developed by Ware and co-workers [20-24]. SF-36 consists of 36 questions divided over eight subscales, covering both physical and mental health. Physical health subscales are Physical functioning (PF), Role physical functioning (RP), Bodily pain (BP) and General health $(\mathrm{GH})$; mental health subscales are Mental health (MH), Role emotional functioning (RE), Social functioning (SF) and Vitality (VT). Questions are stated in either yes/no form or multiple choice. Sum score is calculated for each subscale and transformed to a percentage of the total possible score. A high score indicates good QOL, while a low score indicates lower QOL. The reliability in two general population samples as reported by Aaronson using Cronbach's $\alpha$ varied from 0.76 to 0.92 , while the item discriminant validities varied from 0.09 to 0.64 .

The RQLQ developed by Juniper and Guyatt [1] was used to estimate the severity of complaints caused by AR. Patients had to score 28 items comprising the following domains: Nasal (NS) symptoms (four items), Practical problems (PP, three items), Non-nasal (NN) symptoms (seven items), Sleep (disorders) (SL, three items), (impairment of) Activities (Act, three items), Eye symptoms (ES, four items) and Emotions (EM, four items). They had to estimate their degree of impairment on a 7 -point scale $(0=$ not bothered, $6=$ extremely bothered).

SF-36 and RQLQ were administered to the patient before tests were performed (e.g. the RQLQ was filled in prior to nasal provocation with HDM). Trained personnel instructed the patient according to the guidelines, defined by the designers of the questionnaires. All patients filled in their questionnaires in a private room without the presence of other persons.

As nasal complaints can be biased by use of medication, all nasal medication was stopped. Oral antihistaminics were stopped 17 days and nasal sprays 4-6 weeks before baseline and follow-up visits to ensure a long enough wash-out period. Patients were allowed to use acrivastine, $8 \mathrm{mg} \mathrm{1-3} \mathrm{times} \mathrm{a} \mathrm{day,}$ but the use of rescue medication was discouraged.

\section{Statistical analysis}

Baseline variables for the impermeable cover group and nonimpermeable cover group were calculated with $\chi^{2}$ test for categorical variables and ANOVA for continuous data.

Analysis of the relationship between RQLQ and SF-36 was performed on the pooled data of the patients by means of canonical correlations for continuous data [25-27]. A condition of proper use of canonical correlation is that the variables multivariately have a normal distribution. Secondly, linearity is assumed in canonical analysis. Finally, it is important that the variables within and across each set are not too highly intercorrelated. Squared multiple correlations

Table 1. Inclusion and exclusion criteria

\begin{tabular}{|c|c|}
\hline Inclusion criteria & Exclusion criteria \\
\hline 1. Age $8-50$ years & 1. Pets at home and positive skin test (index $\geqslant 0.7$ ) and/or RAST $\geqslant 2$ for the pet \\
\hline 2. Not pregnant or lactating & 2. Daily use of inhalation steroids $\geqslant 1600 \mathrm{mcg} / \mathrm{day}$ (adults) or $\geqslant 800 \mathrm{mcg} / \mathrm{day}$ (children) \\
\hline 3. No encasings or willing to remove them for the period of the study & 3. Daily use of oral steroids \\
\hline 4. Clinical history of $A R$ and a positive nasal provocation test with HDM & 4. Daily use of cyclosporine \\
\hline 5. RAST $\geqslant 2[1]$ and/or i.c. skin test index [2] $\geqslant 0.7$ for HDM & 5. Regular use of antibiotics for upper or lower airway infection \\
\hline 6. $\geqslant 200 \mathrm{ng}$ Der $p 1$ or Der $f 1$ in dust sample of mattress & 6. Regular use of oral steroids for exacerbation of asthma \\
\hline
\end{tabular}

AR, allergic rhinitis; HDM, house dust mite; i.c., intracutaneous. 
as a measure to identify multicollinearity and singularity were performed. Our data met the aforementioned criteria. BMDP Statistical Software was used for all analyses.

We wanted to investigate the relationship between SF36 and RQLQ in terms of 'explained variance'. Explained variance is that part of the total variance of a variable that can be estimated or predicted by another variable. For example, if the explained variance of 'NS complaints' by PF is 0.40 then $40 \%$ of the variance of 'NS complaints' can be predicted by PF. Otherwise stated, the overlap between 'NS complaints' and $\mathrm{PF}$ is $40 \%$.

Analysis was performed on the sumscores of the domains of both questionnaires. Also, overlap between SF-36 and RQLQ was calculated by means of redundancy analysis. The redundancy is the average explained variance of the domains of one questionnaire multiplied by a factor called the canonical correlation squared.

\section{Results}

\section{General results}

At baseline, 224 patients were included in the study; 183 patients completed the study. Data eligible for analysis were available for 160 patients. Loss to follow-up was not significantly different between the placebo (17) and intervention group (24) as were the reasons for leaving the study (data not shown). At baseline, biographic data, severity of atopic symptoms (assessed by visual analogue scale, SF-36, RQLQ) and sensitization (assessed by RAST, skin tests, eosinophils) were not significantly different in the placebo and intervention group (data not shown). After 12 months, SF-36 and RQLQ subscale scores were not significantly different in both groups as well (data not shown).

\section{Redundancy analysis}

Tables $2 \mathrm{a}$ and $\mathrm{b}$ show the intercorrelation of SF-36 and RQLQ. At baseline, VT and NN symptoms show considerable overlap ( -0.60$)$. After 12 months, NN symptoms show a more or less similar overlap with PF, RP, GH and VT. VT also has a moderate overlap with EM after 12 months. The results of the explained variance analysis (data not shown) showed that the domain of NN complaints of the RQLQ had a moderate overlap of $44 \%$ with SF-36. After 12 months, the overlap was $57 \%$. At baseline, the domain VT of the SF-36 had a moderate overlap with RQLQ of $40 \%$; after 12 months this percentage was $48 \%$. The domain RP of SF-36 had little overlap at baseline with RQLQ, but after 12 months the explained variance was $41 \%$. The overall redundancies for RQLQ and SF-36 subscales were $16 \%$ and $22 \%$, respectively, at baseline. One year after the start of the study, these percentages were 25 for RQLQ and 27 for SF-36 (Table 3).

\section{Discussion}

QOL questionnaires attempt to estimate the burden of disease. In AR, both the generic SF-36 and the diseasespecific RQLQ have often been used [7-9, 28, 29, 1, 13, 30,
Table 2. Intercorrelation matrix of RQLQ and SF-36

\begin{tabular}{llllllll}
\hline & Act & SL & NN & PP & NS & ES & EM \\
\hline (a) At baseline & & & & & & & \\
PF & -0.15 & -0.26 & -0.36 & -0.02 & -0.13 & -0.18 & -0.19 \\
RP & -0.23 & -0.37 & -0.49 & -0.18 & -0.28 & -0.25 & -0.34 \\
GH & -0.10 & -0.30 & -0.46 & -0.06 & -0.19 & -0.25 & -0.38 \\
BP & -0.23 & -0.33 & -0.38 & -0.02 & -0.11 & -0.17 & -0.35 \\
SF & -0.16 & -0.30 & -0.46 & -0.07 & -0.13 & -0.27 & -0.37 \\
VT & -0.20 & -0.33 & -0.60 & -0.13 & -0.25 & -0.33 & -0.46 \\
MH & -0.07 & -0.24 & -0.36 & -0.04 & -0.15 & -0.24 & -0.39 \\
RE & -0.02 & -0.12 & -0.30 & 0.02 & -0.13 & -0.13 & -0.27 \\
(b) After 12 months & & & & & & \\
PF & -0.31 & -0.37 & -0.58 & -0.27 & -0.26 & -0.29 & -0.36 \\
RP & -0.37 & -0.41 & -0.62 & -0.25 & -0.34 & -0.32 & -0.46 \\
GH & -0.29 & -0.30 & -0.52 & -0.28 & -0.25 & -0.37 & -0.42 \\
BP & -0.29 & -0.35 & -0.44 & -0.19 & -0.18 & -0.21 & -0.30 \\
SF & -0.26 & -0.38 & -0.41 & -0.28 & -0.27 & -0.28 & -0.40 \\
VT & -0.35 & -0.47 & -0.69 & -0.34 & -0.38 & -0.36 & -0.51 \\
MH & -0.25 & -0.24 & -0.43 & -0.22 & -0.19 & -0.27 & -0.42 \\
RE & -0.14 & -0.17 & -0.28 & -0.11 & -0.12 & -0.08 & -0.23 \\
\hline
\end{tabular}

Act, (impairment of) Activities (Act, $n=3$ ); BP, Bodily pain; EM, Emotions; ES, Eye symptoms; GH, General health; MH, Mental health; NN, Non-nasal symptoms; NS, Nasal symptoms; PF, Physical functioning; PP, Practical problems; RE, Role emotional functioning; RP, Role physical functioning; SF, Social functioning; SL, Sleep (disorders); VT, Vitality.

Table 3. Overall redundancy of RQLQ and SF-36 (pooled data)

\begin{tabular}{lll}
\hline Canonical variate & RQLQ & SF-36 \\
\hline & Baseline & \\
1 & 0.16 & 0.22 \\
2 & 0.01 & 0.01 \\
& 12 months & \\
1 & 0.25 & 0.27 \\
2 & 0.02 & 0.01 \\
\hline
\end{tabular}

Redundancy coefficients: the percentage of variance extracted from its own set of variables multiplied by the canonical correlation squared for the pair. Only the first pair was of significance.

31]. It has been stated that generic instruments are more tailored to evaluate the burden of disease across different diseases, whereas specific instruments are more responsive for changes in clinical trials [32]. Although both instruments aim to evaluate QOL, it is not known whether these instruments cover similar domains rather than different domains in QOL. It is possible that some domains are covered better by one instrument than by the other.

Recently, Juniper et al. reported a moderate correlation between SF-36 and RQLQ [33]. The subjects tested were not permitted to have symptoms other than those of rhinoconjunctivitis. By focussing on subjects with rhinitis only, this investigation gives a clear insight in the inter-relationship between the two instruments by studying a homogeneous patient population. It is, however, well known that rhinitis, asthma and AD are closely associated. Atopy - the personal or familial tendency to produce IgE antibodies to low-dose antigens and to develop typical symptoms such as asthma, rhinoconjunctivitis and $\mathrm{AD}$ - forms the basis of the close 
connection between these disorders [34]. Development of allergic sensitization and diseases is partly under common genetic control. Evidence for linkage between candidate loci for allergic disease and total IgE concentrations is found in the same regions for which reasonable evidence for linkage to asthma has been reported [35].

The patient population of this study reflects the close association between AR, asthma and AD as shown in a comorbidity percentage of $53 \%$ (data not shown). An exploration of the inter-relation of SF-36 and RQLQ in this study group as opposed to the rhinoconjunctivitis group of Juniper was therefore a logical consequential step. The explained variance analysis showed an overlap in the domains ' $\mathrm{NN}$ complaints' of the RQLQ and VT of the SF-36; in the intercorrelation matrix, 'NN complaints' also showed an overlap with VT at baseline and after 12 months. It is not surprising that these items of the RQLQ (i.e. being troubled by fatigue, thirst, reduced productivity, tiredness, poor concentration, headache and being worn out as a consequence of nose and eye symptoms) and the SF-36 VT domain (comprising questions as 'Did you feel full of pep?', 'Did you have a lot of energy?', 'Did you feel worn out?' and 'Did you feel tired?') can be explained by the other instrument. The same may hold true for the overlap of 'NN complaints' after 12 months with PF, RP and GH. Other domains of the SF-36 apparently have little overlap with the domains of the RQLQ and vice versa. Overall redundancy coefficients were low, 0.16 and 0.21 for RQLQ and SF-36, respectively.

The overlap may be partly explained by our observation that associated NN symptoms (i.e. asthma symptoms and SL disorders) or disease (presence of asthma) influence generic QOL [17]. That the redundancy is moderate can be partly attributed to the fact that the measurements are fallible. It had already been shown in the 1970s by Andrew and Withey that a subjective QOL judgement contains about $60 \%$ valid variance. The remaining variance comprises both the systematic and the random error variance.

In our analysis, we used the pooled data of the patients under the assumption that a possible effect of the intervention would affect SF-36 and RQLQ equally. However, it is conceivable that the responsiveness of a disease-specific instrument is better compared with a generic instrument. As we could not demonstrate any effect of the covers on other clinical end points [19] despite a significant reduction in exposure to allergen, we find it improbable that such a difference in responsiveness would have appeared.

The results of questionnaires might be biased because of errors of the measurement instrument such as distortion by the format of questionnaire design or a patient's disposition to give socially desirable answers, to represent themselves as better than they actually feel (faking good) or the opposite (faking bad). A patient's frame of references can change considerably over time, as is known from oncology research, $[36,37]$ and this response shift has to be taken into account in all QOL research ranging over time.

However, neither the overlap between both instruments changed substantially in 12 months, nor was a significant change in scores on SF36 and RQLQ subscales observed. These results are in line with our observation that the use of impermeable covers for bedding does not have a beneficial effect on AR [19], which excludes a differential effect of treatment on the outcome of both instruments. Although patients were monitored during 12 months, the findings do not suggest a response shift.

The limited overlap between RQLQ and SF-36 may have some consequences. The burden of rhinitis and the effect of a pharmacological intervention focussed on rhinitis may be best evaluated by a disease-specific questionnaire such as the RQLQ. Recognizing the fact that AR forms a part of the atopic syndrome and comes with other atopic disorders, AR in the context of co-morbid associations may be best evaluated by a generic instrument such as the SF-36. Therefore, the use of both types of instruments simultaneously may be advocated in the evaluation of the patient with AR.

In conclusion, in patients with HDM allergy characterized by co-morbid associations SF-36 and RQLQ cover different aspects in QOL and should be used simultaneously in performing QOL studies.

\section{Acknowledgements}

This project was supported by the Dutch Asthma Foundation, project number 32.98.14. The project was part of Dutch Mite Avoidance Study (DUMAS). We are indebted to Miss J. H. Broeshart, MD, Miss S. H. Hendriks, MD, Mrs A. J. van Oorschot-van Nes, Miss L. Havekes and Mrs D. van der Naald for their invaluable contribution to the project.

We also wish to thank $\mathrm{H}$. de Groot, $\mathrm{MD}, \mathrm{PhD}$, for his valuable comments on earlier versions of this article.

\section{References}

1 Juniper EF, Guyatt GH. Development and testing of a new measure of health status for clinical trials in rhinoconjunctivitis. Clin Exp Allergy 1991; 21:77-83.

2 Juniper EF, Guyatt GH, Epstein RS, Ferrie PJ, Jaeschke R, Hiller TK. Evaluation of impairment of health related quality of life in asthma: development of a questionnaire for use in clinical trials. Thorax 1992; 47:76-83.

3 Molen van der T, Postma DS, Schreurs AJ, Bosveld HE, Sears MR, Meyboom de Jong B. Discriminative aspects of two generic and two asthma-specific instruments: relation with symptoms, bronchodilator use and lung function in patients with mild asthma. Qual Life Res 1997; 6:353-61.

4 Bousquet J, Knani J, Dhivert $\mathrm{H}$ et al. Quality of life in asthma. I. Internal consistency and validity of the SF-36 questionnaire. Am J Respir Crit Care Med 1994; 149:371-5.

5 Finlay AY, Khan GK. Dermatology Life Quality Index (DLQI) a simple practical measure for routine clinical use. Clin Exp Dermatol 1994; 19:210-6.

6 Baiardini I, Pasquali M, Giardini A et al. Rhinasthma: a new specific QoL questionnaire for patients with rhinitis and asthma. Allergy 2003; 58:289-94.

7 Bousquet J, Duchateau J, Pignat JC et al. Improvement of quality of life by treatment with cetirizine in patients with perennial allergic rhinitis as determined by a French version of the SF-36 questionnaire. J Allergy Clin Immunol 1996; 98:309-16.

8 Bousquet J, Bullinger M, Fayol C, Marquis P, Valentin B, Burtin B. Assessment of quality of life in patients with perennial allergic rhinitis with the French version of the SF-36 Health Status Questionnaire. J Allergy Clin Immunol 1994; 94:182-8. 
9 Pariente PD, LePen C, Los F, Bousquet J. Quality-of-life outcomes and the use of antihistamines in a French national populationbased sample of patients with perennial rhinitis. Pharmacoeconomics 1997; 12:585-95.

10 Juniper EF, Guyatt GH, O'Byrne PM, Viveiros M. Aqueous beclomethasone dipropionate nasal spray: regular vs. "as required" use in the treatment of seasonal allergic rhinitis. J Allergy Clin Immunol 1990; 86:380-6.

11 Juniper EF, Willms DG, Guyatt GH, Ferrie PJ. Aqueous beclomethasone dipropionate nasal spray in the treatment of seasonal (ragweed) rhinitis. Cmaj 1992; 147:887-92.

12 Kremer B, Klimek L, Bullinger M, Mosges R. Generic or diseasespecific quality of life scales to characterize health status in allergic rhinitis? Allergy 2001; 56:957-63.

13 de Graaf-in 't Veld T, Koenders S, Garrelds IM, Gerth van Wijk R. The relationships between nasal hyperreactivity, quality of life, and nasal symptoms in patients with perennial allergic rhinitis. J Allergy Clin Immunol 1996; 98:508-13.

14 Edfors-Lubs ML. Allergy in 7000 twin pairs. Acta Allergol 1971; 26:249-85.

15 Peckham C, Butler N. A national study of asthma in childhood. J Epidemiol Community Health 1978; 32:79-85.

16 Leynaert B, Neukirch F, Demoly P, Bousquet J. Epidemiologic evidence for asthma and rhinitis comorbidity. J Allergy Clin Immunol 2000; 106:201-5.

17 Terreehorst I, Oosting AJ, Tempels-Pavlica Z et al. Prevalence and severity of allergic rhinitis in house dust mite-allergic patients with bronchial asthma or atopic dermatitis. Clin Exp Allergy 2002; $32: 1160-5$.

18 Terreehorst I, Duivenvoorden HJ, Tempels-Pavlica Z et al. The unfavorable effects of concomitant asthma and sleeplessness due to the atopic eczema/dermatitis syndrome (AEDS) on quality of life in subjects allergic to house-dust mites. Allergy 2002; 57:919-25.

19 Terreehorst I, Hak E, Oosting AJ et al. Evaluation of impermeable covers for bedding in patients with allergic rhinitis. N Engl J Med 2003; 349:237-46.

20 Aaronson NK, Muller M, Cohen PD et al. Translation, validation, and norming of the Dutch language version of the SF-36 Health Survey in community and chronic disease populations. J Clin Epidemiol 1998; 51:1055-68.

21 Keller SD, Bayliss MS, Ware JE Jr, Hsu MA, Damiano AM, Goss TF. Comparison of responses to SF-36 Health Survey questions with oneweek and four-week recall periods. Health Serv Res 1997; 32:367-84.

22 Ware JE Jr, Sherbourne CD. The MOS 36-item short-form health survey (SF-36). I. Conceptual framework and item selection. Med Care 1992; 30:473-83.
23 Ware JE Jr, Keller SD, Gandek B, Brazier JE, Sullivan M. Evaluating translations of health status questionnaires. Methods from the IQOLA project. International Quality of Life Assessment. Int J Technol Assess Health Care 1995; 11:525-51.

24 Ware JE Jr, Kemp JP, Buchner DA, Singer AE, Nolop KB, Goss TF. The responsiveness of disease-specific and generic health measures to changes in the severity of asthma among adults. Qual Life Res 1998; 7:235-44.

25 Morrison D. Multivariate statistical methods. New York: McGraw-Hill, 1967.

26 Anderson TW. Introduction to multivariate statistical analysis. New York: Wiley, 1958.

27 Cooley W, Lohnes P. Multivariate data analysis. New York: Wiley, 1971.

28 Leynaert B, Neukirch C, Liard R, Bousquet J, Neukirch F. Quality of life in allergic rhinitis and asthma. A population-based study of young adults. Am J Respir Crit Care Med 2000; 162:1391-6.

29 Meltzer EO. Quality of life in adults and children with allergic rhinitis. J Allergy Clin Immunol 2001; 108:S45-53.

30 Van Cauwenberge P, Juniper EF. Comparison of the efficacy, safety and quality of life provided by fexofenadine hydrochloride $120 \mathrm{mg}$, loratadine $10 \mathrm{mg}$ and placebo administered once daily for the treatment of seasonal allergic rhinitis. Clin Exp Allergy 2000; 30:891-9.

31 Ellis AK, Day JH, Lundie MJ. Impact on quality of life during an allergen challenge research trial [In Process Citation]. Ann Allergy Asthma Immunol 1999; 83:33-9.

32 Guyatt GH, King DR, Feeny DH, Stubbing D, Goldstein RS. Generic and specific measurement of health-related quality of life in a clinical trial of respiratory rehabilitation. J Clin Epidemiol 1999; 52:187-92.

33 Juniper EF, Thompson AK, Roberts JN. Can the standard gamble and rating scale be used to measure quality of life in rhinoconjunctivitis? Comparison with the RQLQ and SF-36. Allergy 2002; 57:201-6.

34 Johansson SG, Hourihane JO, Bousquet $\mathbf{J}$ et al. A revised nomenclature for allergy. An EAACI position statement from the EAACI nomenclature task force. Allergy 2001; 56:813-24.

35 Barnes KC. Evidence for common genetic elements in allergic disease. J Allergy Clin Immunol 2000; 106:S192-200.

36 Visser MR, Smets EM, Sprangers MA, de Haes HJ. How response shift may affect the measurement of change in fatigue. J Pain Symptom Manage 2000; 20:12-8.

37 Sprangers MA, de Regt EB, Andries F et al. Which chronic conditions are associated with better or poorer quality of life? [In Process Citation]. J Clin Epidemiol 2000; 53:895-907. 